\title{
Desempenho motor em uma amostra de escolares brasileiros
}

\author{
Motor performance in a sample of Brazilian schoolchildren
}

\author{
D.P. Guedes, J.T.M. Neto, A.J. Silva
}

RESUMO

O estudo analisou o desempenho motor em relação à idade cronológica e ao sexo em amostra representativa de escolares de Montes Claros, Minas Gerais, Brasil. A amostra foi constituída por 2849 sujeitos (1457 raparigas e 1392 rapazes), com idades entre 6 e 18 anos, selecionada aleatoriamente. Os componentes de desempenho motor foram determinados a partir da bateria de teste envolvendo cinco itens: a) sit-and-reach, b) curl-ups, c) trunk-lift, d) push-ups e e) PACER. Os resultados encontrados mostram que, em quase todos os testes motores administrados foram constatadas diferenças intersexos desde os 6 anos que aumentaram drasticamente a partir dos 12-13 anos. Em relação à idade cronológica, os rapazes na maioria dos testes motores administrados apresentaram gradualmente melhores resultados desde os 6 até os 18 anos, enquanto entre as raparigas os resultados mais elevados ocorreram por volta dos 11-12 anos com posterior declínio ou constância de seus escores.

Palavras-chave: testes motores, aptidão física, promoção de saúde, criança, adolescente

This study analyzed the analyzed the relationship among motor performance, age and gender in a representative sample of schoolchildren from Montes Claros, Minas Gerais, Brazil. The sample consisted of 2849 subjects (1457 girls and 1392 boys), ranging in age from 6 to 18 years, selected randomly. The motor performance components were determined using a test battery consisting of five items: a) sit-and-reach, b) curl-ups, c) trunk-lift, d) push-ups, and e) PACER. The results show that, in almost all motor tests occurred differences intersexes from 6 years and up which increased abruptly after 12-13 years. In relation to chronological age, boys presented gradually better results from 6 to 18 years in the majority of the motor tests. Girls' best performance occurred around 11-12 years of age followed by a steady performance or a tendency to decline.

Keywords: motor tests, physical fitness, health promotion, children, adolescents

Dartagnan Pinto Guedes. Universidade Estadual de Londrina, Paraná, Brasil.

Jaime Tolentino Miranda Neto. Grupo Integrado de Pesquisa em Psicologia do Esporte/Exercício e Saúde, Saúde Ocupacional e Mídia - GIPESOM; Universidade Estadual de Montes Claros, Minas Gerais, Brasil.

António José Rocha Martins da Silva. Centro de Investigação em Desporto, Saúde e Desenvolvimento Humano CIDESD; Universidade de Trás-os-Montes e Alto Douro, Vila Real, Portugal.

Endereço para correspondência: Dartagnan Pinto Guedes, Rua Ildefonso Werner, 177, Condomínio Royal Golf, CEP: 86055-545 - Londrina, Paraná, Brasil.

E-mail:darta@sercomtel.com.br 
A literatura oferece convictas evidências no sentido de que a prática suficiente e adequada de atividade física induz importantes benefícios para a saúde dos jovens (Bauman, 2004; Hallal, Victora, Azevedo, \& Wells, 2006), e hábitos sedentários na infância e na adolescência tendem a permanecer em idades adultas (Gordon-Larsen, Nelson, \& Popkin, 2004; Telama et al., 2005). A despeito das evidências, atualmente, jovens de diferentes sociedades vêm demonstrado serem menos ativos fisicamente que em décadas recentes (Allison, Adlaf, Dwyer, Iysy, \& Irving, 2007; Hardy, Okely, Dobbins, \& Booth, 2008). Provavelmente, justifica-se o declínio nos níveis de prática de atividade física em razão das inúmeras escolhas que os jovens têm disponível para o seu tempo de lazer. Televisão, jogos eletrônicos, internet e outras atividades sedentárias têm ocupado muito tempo do jovem em substituição a outras atividades de lazer que exigem algum esforço físico (Nelson, Neumark-Stzainer, Hannan, Sirard, \& Story, 2006). A redução do tempo destinado aos programas de educação física escolar e atividades envolvendo esforços físicos de menor intensidade nas aulas também contribui para o aumento da prevalência de sedentarismo em populações jovens (Fairclough \& Stratton, 2006).

A proficiência quanto ao desempenho motor caracteriza-se como um importante atributo no repertório de conduta motora em idades jovens, tornando-se, portanto, essencial para a efetiva adoção de um estilo de vida ativo fisicamente (Malina, Bouchard, \& Bar-Or, 2004). Por essa razão, mais recentemente tem surgido considerável interesse no desenvolvimento de estudos direcionados a reunir informações voltadas ao desempenho motor de integrantes de populações jovens de diferentes contextos ambientais e socioculturais (Freitas et al., 2002; Jürimäe \& Volbekiene, 1998; Malina, 2007; Mondal, 2006; Özdirenç, Özcan, Akin, \& Gelecek, 2005; Pena-Reys, Tan, \& Malina, 2003; Tinazci \& Emiroglu, 2009). Ainda, considerando que a infância e a adolescência se constituem no período crítico mais importante associado ao campo motor, seja em relação aos componentes biológicos como aos componentes comportamentais, no qual o organismo jovem se encontra especialmente sensível à influência de fatores tanto de natureza positiva como negativa, o levantamento de informações voltadas ao desempenho motor, nesse período, poderá contribuir decisivamente na tentativa de promoção da saúde coletiva.

Nessa perspectiva, o objetivo do estudo foi reunir informações que possa traduzir o comportamento de escores de testes motores que evidenciam características de desempenho motor em relação ao género e à idade cronológica da população escolar da cidade de Montes Claros, Minas Gerais, Brasil.

\section{MÉTODO}

Trata-se de um levantamento descritivo de corte transversal com base populacional. A coleta de dados foi realizada de abril a novembro de 2007. Os protocolos de intervenção utilizados foram aprovados pelo Comitê de Ética em Pesquisa da Universidade Estadual de Montes Claros, Brasil, e acompanharam normas e diretrizes regulamentadoras de pesquisa envolvendo seres humanos.

A cidade de Montes Claros, cenário do presente estudo, localiza-se no norte do estado de Minas Gerais, Brasil. Para sua caracterização, assumindo como referência o Índice de Desenvolvimento Humano (IDH), verifica-se que a cidade de Montes Claros, nas duas últimas décadas, vem apresentando importante evolução. Contudo, ainda assim, apresenta IDH menor que as cidades de similar densidade populacional localizadas em outras regiões brasileiras, sobretudo, da região sudeste e sul. Como ilustração, no período de 1991-2006, o IDH de Montes Claros cresceu 10\%, passando de $.713 \mathrm{em} 1991$ para $.783 \mathrm{em}$ 2006. A dimensão que mais contribuiu para este crescimento foi a educação (.793 versus .872) seguida da renda (.629 versus .681) e da 
longevidade (.741 versus .787). Neste período, o hiato de desenvolvimento humano, ou seja, a distância entre o IDH do município e o limite máximo de IDH (1 - IDH), foi reduzido em $32 \%$. O IDH-2006 médio das cidades localizadas nas regiões sudeste e sul foi $.844 \mathrm{e}$ .825 , respectivamente, e o IDH-2006 brasileiro foi .800 (PNUD, 2007).

\section{Amostra}

A população de referência para o estudo incluiu os escolares de ambos os géneros, entre os 6 e os 18 anos de idade, regularmente matriculados no ano letivo de 2007, nas escolas de ensino público e privado do município de Montes Claros, Minas Gerais, Brasil. Para ilustrar a dimensão do universo populacional tratado, de acordo com informações do Setor de Estatística da Secretaria da Educação do Estado de Minas Gerais, estavam matriculados no início do ano letivo de 2007, nas 237 escolas localizadas na região urbana do município, por volta de 85 mil escolares.

O tamanho da amostra foi estabelecido assumindo um intervalo de confiança de $95 \%$, um erro amostral de $3 \%$ e um acréscimo de $10 \%$ para atender eventuais casos de perdas na coleta dos dados. Considerando que o planejamento amostral envolveu conglomerados, definiu-se um efeito do delineamento da amostra equivalente a 1.5, prevendo inicialmente uma amostra mínima de 2600 escolares. Porém, a amostra definitiva utilizada no tratamento das informações foi composta por 2849 escolares (1457 raparigas e 1392 rapazes).

Quanto à seleção dos escolares, foi utilizada uma amostragem probabilística por conglomerados, tendo como referência o número de escolares quanto ao género e à série em que estavam matriculados em cada estrato da estrutura escolar (pública e privada). Assim, a primeira etapa foi determinar a representatividade do número de escolares de cada estrato da estrutura escolar em relação a toda a população estudada. Depois, a representatividade do número de escolares matriculados em cada escola selecionada para estudo em relação à população escolar do estrato da estrutura escolar a que pertence. Para a seleção dos escolares em cada escola, inicialmente foi realizado um sorteio de turmas constituídas especificamente para as sessões de educação física e, posteriormente, dentro de cada turma selecionada, foram sorteados os escolares necessários para compor a amostra representativa da escola. Os critérios adotados para a exclusão de algum escolar sorteado para o estudo foram: i) recusa em participar do estudo; ii) não autorização dos pais ou responsáveis; iii) ausência às aulas no dia agendado para a coleta dos dados; e, iv) algum problema físico que o impedisse, temporária ou definitivamente, de realizar os testes motores.

\section{Instrumentos e Procedimentos}

Os componentes associados ao desempenho motor foram analisados mediante escores equivalentes aos resultados observados por meio da aplicação de bateria de testes motores composta por cinco itens, obedecendo à sequência: a) back-saver sit-and-reach; b) curl-ups; c) trunk-lift; d) push-ups; e, e) progressive endurance run (PACER), descritos originalmente na proposta sugerida pelo FitnessGram (CIAR, 1999).

A idade cronológica dos escolares foi determinada de forma centesimal, a partir da confrontação entre a data de coleta dos dados e a data de nascimento. Para a formação dos grupos etários, a idade inferior foi considerada em .50 e a idade superior em .49 , centralizando a idade intermediária em anos completos. Como ilustração, o grupo etário de 10 anos foi formado tomando-se esta idade como posição intermediária e agrupando-se as informações dos 9.50 até 10.49 anos de idade.

A equipe de avaliadores foi composta por quatro profissionais de educação física e oito acadêmicos do último ano do curso de educação física, supervisionados pelos próprios autores. Anteriormente ao início da coleta dos 
dados, os avaliadores responsáveis pela tomada dos dados foram submetidos a um período de treinamento na tentativa de minimizar ao máximo a influência de viés intra-avaliadores. Além disso, cada um dos avaliadores desempenhou sempre a mesma função durante todo o processo de coleta dos dados. Os testes motores foram aplicados em uma única sessão de coleta dos dados.

\section{Análise Estatística}

O tratamento estatístico dos dados foi realizado mediante o pacote computadorizado Statistical Package for the Social Science (SPSS), versão 17.0.

Os dados foram inicialmente comparados com a curva normal utilizando o teste de distância K-S (Kolmogorov-Smirnov). Considerando que os dados mostraram distribuição de frequência normal, recorreu-se aos recursos da estatística paramétrica, mediante cálculo de média e desvio-padrão e, posteriormente, para detecção de eventuais diferenças entre género e idade utilizou-se da análise de variância (ANOVA) do tipo two-way, acompanhada do teste post-hoc de comparação múltipla de Scheffé para identificação de diferenças específicas, quando os valores de $F$ encontrados mostraram-se superiores ao critério de significância pré-estabelecido em $.01<p<.05$ e $p \leq .01$.

\section{RESULTADOS}

As tabelas 1 e 2 mostram informações relacionadas aos escores equivalentes aos resultados dos testes motores.

Em geral, os rapazes demonstram escores mais elevados a cada ano desde os 6 até os 18 anos de idade, enquanto as raparigas apresentam, na maioria das vezes, resultados mais elevados nos testes motores somente até por volta dos 12-13 anos.

Tabela 1

Análise comparativa por género dos escores equivalentes aos resultados de testes motores (sit-and-reach, curl-ups e trunk-lift) de escolares de Montes Claros, Minas Gerais, Brasil, 2007

\begin{tabular}{ccccccc}
\hline \multirow{2}{*}{$\begin{array}{c}\text { Idade } \\
\text { (Anos) }\end{array}$} & Raparigas & Rapazes & Raparigas & Rapazes & Raparigas & Rapazes \\
& $M \pm D P$ & $M \pm D P$ & $M \pm D P$ & $M \pm D P$ & $M \pm D P$ & $M \pm D P$ \\
\hline 6 & $27.82 \pm 6.41$ & $27.98 \pm 5.87$ & $12.02 \pm 2.48$ & $12.34 \pm 3.54$ & $20.51 \pm 4.55$ & $20.47 \pm 4.25$ \\
7 & $27.50 \pm 4.68$ & $27.81 \pm 4.54$ & $13.58 \pm 3.62$ & $13.63 \pm 3.75$ & $21.31 \pm 5.48$ & $21.05 \pm 4.84$ \\
8 & $26.91 \pm 3.97$ & $27.55 \pm 5.09$ & $14.79 \pm 3.50$ & $15.20 \pm 3.81$ & $23.19 \pm 5.83$ & $22.81 \pm 4.68$ \\
9 & $26.31 \pm 5.53$ & $26.95 \pm 4.89$ & $16.13 \pm 4.34$ & $16.28 \pm 4.14$ & $23.49 \pm 5.28$ & $23.15 \pm 6.19$ \\
10 & $25.58 \pm 5.53$ & $26.35 \pm 5.08$ & $17.86 \pm 4.64$ & $18.68 \pm 5.59$ & $23.79 \pm 4.64$ & $24.17 \pm 5.48$ \\
11 & $24.84 \pm 5.91$ & $24.99 \pm 5.40$ & $18.87 \pm 5.23$ & $20.09 \pm 6.31$ & $24.54 \pm 5.79$ & $25.01 \pm 6.13$ \\
12 & $25.22 \pm 6.42$ & $24.79 \pm 5.64$ & $20.23 \pm 7.61$ & $23.12 \pm 7.31$ & $25.43 \pm 6.47$ & $25.85 \pm 6.77$ \\
13 & $25.59 \pm 7.05$ & $24.56 \pm 6.44$ & $20.86 \pm 8.43$ & $25.45 \pm 7.39^{*}$ & $25.95 \pm 5.97$ & $25.51 \pm 6.33$ \\
14 & $26.02 \pm 6.82$ & $25.38 \pm 6.35$ & $21.83 \pm 8.61$ & $27.77 \pm 8.74^{*}$ & $24.58 \pm 5.98$ & $25.45 \pm 6.23$ \\
15 & $26.85 \pm 6.45$ & $26.29 \pm 7.57$ & $21.84 \pm 8.41$ & $30.14 \pm 9.20^{*}$ & $24.07 \pm 5.67$ & $25.23 \pm 7.05$ \\
16 & $27.68 \pm 7.08$ & $27.16 \pm 6.16$ & $21.40 \pm 7.71$ & $31.59 \pm 9.62^{* *}$ & $24.13 \pm 8.64$ & $24.70 \pm 6.57$ \\
17 & $28.48 \pm 6.83$ & $27.89 \pm 7.78$ & $20.64 \pm 7.29$ & $32.95 \pm 8.68^{* *}$ & $24.19 \pm 6.51$ & $24.19 \pm 5.87$ \\
18 & $28.83 \pm 8.14$ & $28.21 \pm 7.16$ & $19.58 \pm 6.97$ & $33.15 \pm 8.45^{* *}$ & $23.78 \pm 6.75$ & $24.15 \pm 7.41$ \\
\hline$F_{\text {idade }}$ & $2.47(p=.119)$ & $54.58(p<.001)$ & $1.46(p=.173)$ \\
$F_{\text {sexo }}$ & $3.63(p=.057)$ & $127.89(p<.001)$ & $.97(p=.326)$ \\
$F_{\text {interação }}$ & $1.29(p=.219)$ & $11.73(p<.001)$ & $1.00(p=.443)$ \\
\hline Difench $)$ &
\end{tabular}

${ }^{*}$ Diferenças significativas entre géneros $(p<.05) ;{ }^{* *}$ Diferenças significativas entre géneros $(p<.01)$ 
Tabela 2

Análise comparativa por género dos escores equivalentes aos resultados de testes motores (push-ups e PACER) de escolares de Montes Claros, Minas Gerais, Brasil, 2007

\begin{tabular}{|c|c|c|c|c|}
\hline \multirow{3}{*}{$\begin{array}{c}\text { Idade } \\
\text { (Anos) }\end{array}$} & \multicolumn{2}{|c|}{ Push-Ups (rep) } & \multicolumn{2}{|c|}{ PACER (voltas) } \\
\hline & Raparigas & Rapazes & Raparigas & Rapazes \\
\hline & $M \pm D P$ & $M \pm D P$ & $M \pm D P$ & $M \pm D P$ \\
\hline 6 & $2.04 \pm 1.10$ & $3.68 \pm 1.12 *$ & $15.93 \pm 4.95$ & $16.34 \pm 4.29$ \\
\hline 7 & $2.20 \pm 1.37$ & $4.02 \pm 1.28^{*}$ & $17.58 \pm 4.30$ & $19.80 \pm 5.01$ \\
\hline 8 & $2.48 \pm 1.49$ & $4.41 \pm 1.60^{*}$ & $19.23 \pm 6.83$ & $22.67 \pm 7.18$ \\
\hline 9 & $2.89 \pm 1.65$ & $5.39 \pm 1.74^{* *}$ & $20.42 \pm 7.86$ & $23.88 \pm 7.14$ \\
\hline 10 & $3.09 \pm 1.72$ & $5.83 \pm 1.81^{* *}$ & $21.69 \pm 8.02$ & $25.70 \pm 7.88$ \\
\hline 11 & $3.20 \pm 1.78$ & $5.96 \pm 1.92^{* *}$ & $22.14 \pm 8.08$ & $28.53 \pm 8.04^{*}$ \\
\hline 12 & $3.37 \pm 1.67$ & $6.43 \pm 2.27^{* *}$ & $23.12 \pm 7.48$ & $31.35 \pm 9.26^{*}$ \\
\hline 13 & $3.18 \pm 1.41$ & $7.34 \pm 2.76^{* *}$ & $25.08 \pm 8.41$ & $35.79 \pm 9.76^{*}$ \\
\hline 14 & $3.17 \pm 1.79$ & $9.24 \pm 3.75^{* *}$ & $24.91 \pm 8.86$ & $39.24 \pm 9.35^{* *}$ \\
\hline 15 & $3.16 \pm 1.86$ & $11.88 \pm 3.59^{* *}$ & $24.24 \pm 7.82$ & $41.48 \pm 9.46^{* *}$ \\
\hline 16 & $3.09 \pm 1.74$ & $13.54 \pm 3.14^{* *}$ & $24.01 \pm 7.61$ & $43.56 \pm 9.96^{* *}$ \\
\hline 17 & $3.03 \pm 1.17$ & $14.99 \pm 3.29 * *$ & $23.88 \pm 6.59$ & $45.98 \pm 8.09 * *$ \\
\hline 18 & $2.93 \pm 1.13$ & $15.51 \pm 3.75^{* *}$ & $22.22 \pm 6.89$ & $46.42 \pm 8.98^{* *}$ \\
\hline$F_{\text {idade }}$ & \multicolumn{2}{|c|}{$53.10(p<.001)$} & \multicolumn{2}{|c|}{$43.59(p<.001)$} \\
\hline$F_{\text {sexo }}$ & \multicolumn{2}{|c|}{$780.17(p<.001)$} & \multicolumn{2}{|c|}{$341.45(p<.001)$} \\
\hline$F_{\text {interação }}$ & \multicolumn{2}{|c|}{$45.44(p<.001)$} & \multicolumn{2}{|c|}{$17.35(p<.001)$} \\
\hline
\end{tabular}

Em idades mais avançadas, os escores tendem a declinar ou a permanecer constantes. Consequentemente, em ambos os sexos, as curvas representativas dos escores equivalentes aos resultados dos testes motores mostram-se essencialmente ascendentes e paralelas até próximas dos 12-13 anos de idade. Depois, enquanto os escores apresentados pelos rapazes induzem a uma elevação contínua nas inclinações das curvas, sugerindo o aparecimento de um surto de desempenho motor nesse período, entre as raparigas as curvas tendem a ser mais planas, oferecendo indícios de já terem alcançado o estágio adulto quanto ao desempenho motor (ver figura 1).

Naquelas tarefas motoras em que é exigido o deslocamento e a sustentação parcial ou total da massa corporal (curl-ups e push-ups) e o transporte da própria massa corporal mediante caminhada/corrida (PACER), as diferenças entre rapazes e raparigas tornam-se progressivamente maiores com a idade e se acentuam enormemente a partir dos 13-14 anos. Por outro lado, nas tarefas em que a solicitação motora envolve prioritariamente movimentos articulares sem o deslocamento ou o transporte da massa corporal, como é o caso dos testes sit-and-reach e trunk-ups, as diferenças entre ambos os sexos diminui acentuadamente e não são apontadas em linguagem estatística.

\section{DISCUSSÃo}

No presente estudo são apresentados dados acerca de indicadores motores associados à aptidão física relacionada à saúde em integrantes da população escolar do município de Montes Claros, Minas Gerais, Brasil, mediante a utilização da bateria de testes motores FitnessGram. Convém salientar que, 

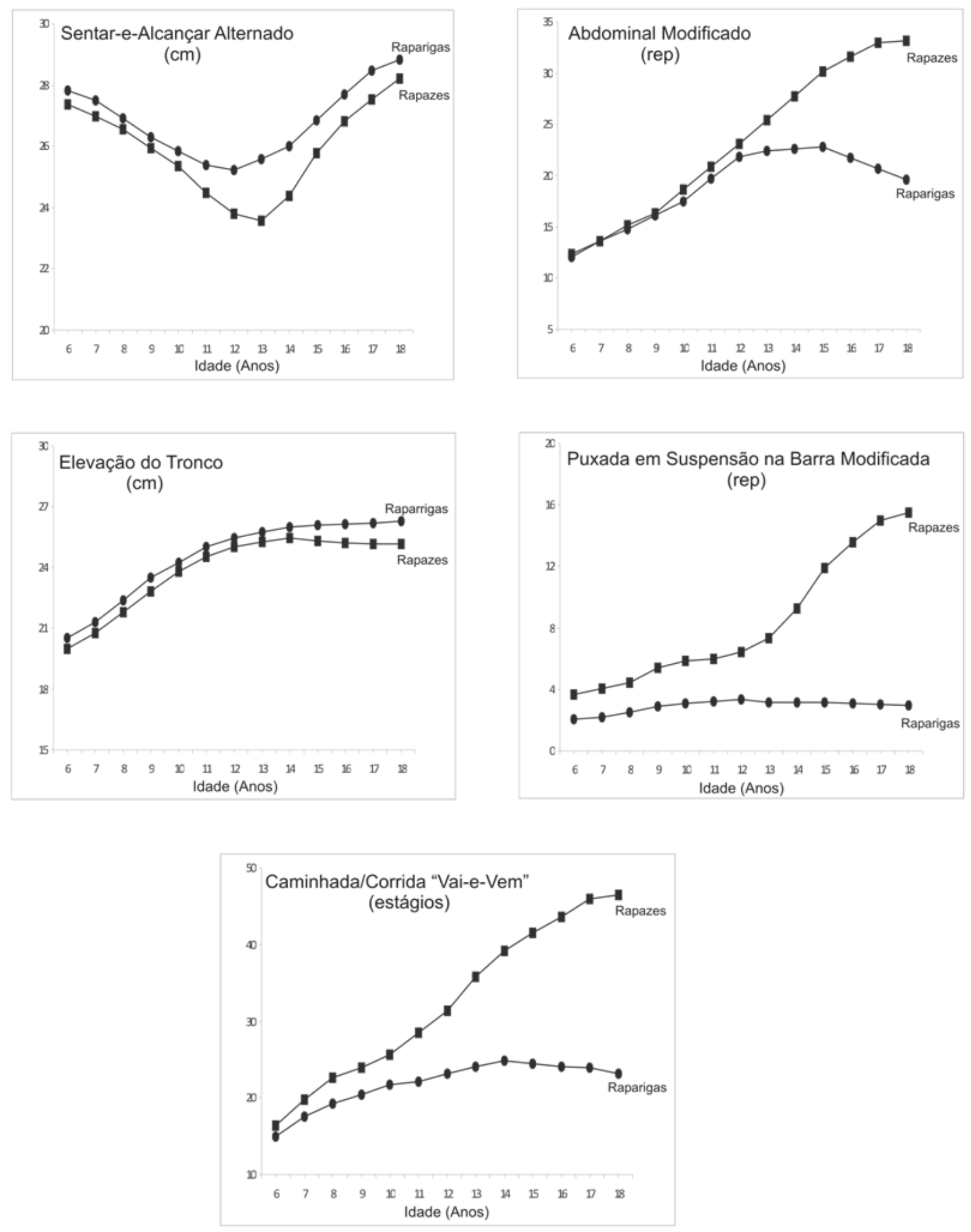

Figura 1. Curvas representativas de escores equivalentes aos resultados de testes motores de escolares de Montes Claros, Minas Gerais, Brasil, 2007 
quando comparadas com informações produzidas mediante recursos laboratoriais, como é o caso da estimativa do consumo máximo de oxigénio, dos valores de força muscular, resistência muscular e mobilidade articular, os escores equivalentes aos resultados de testes motores podem eventualmente apresentar limitações quanto à análise dos componentes da aptidão física relacionada à saúde (Guedes \& Guedes, 2006).

No entanto, apesar das limitações, os escores equivalentes aos resultados de testes motores podem ser extremamente úteis para a análise de indicadores associados aos componentes da aptidão física relacionada à saúde. Neste sentido, a bateria de testes motores FitnessGram, bateria de testes motores empregada neste estudo, vem sendo utilizada em diferentes países com resultados satisfatórios (Fahlman, Hall, \& Lock, 2006; Mota, Flores, Ribeiro, \& Santos, 2006; Kim et al., 2005; Lloyd, Bishop, Walker, Sharp, \& Richardson, 2003; Maia \& Lopes, 2002; Mastrangelo, Chaloupka, \& Rattigan, 2008).

$\mathrm{Na}$ literatura especializada são raros os estudos direcionados à aptidão física relacionada à saúde, de base populacional, envolvendo a população escolar. Além disso, as baterias de testes motores empregadas para análise de seus componentes não apresentam consenso nos estudos já realizados. As distintas definições atribuídas aos critérios de saúde envolvendo escores equivalentes aos resultados de testes motores deve ser considerada outra limitação quando da realização de comparações entre diferentes estudos. Diferenças quanto aos tipos de amostragem também representam limitações para análises comparativas. Portanto, devem-se considerar estas situações ao se estabelecer comparações entre os achados do presente estudo e os resultados disponibilizados para consulta.

Ao analisar os escores equivalentes aos resultados dos testes motores, verificam-se comportamentos em relação às idades bastante divergentes entre os sexos. Os rapazes demonstraram resultados progressivamente mais elevados a cada ano desde os 6 até os 18 anos de idade, evidenciando nitidamente a repercussão positiva que, em um primeiro momento, as modificações estruturais e funcionais que acompanham a maturação biológica podem provocar em seu desempenho motor. As raparigas, por sua vez, demonstraram resultados mais elevados nos testes motores até por volta dos 11-12 anos de idade e, com o advento esperado da puberdade, os escores observados tenderam a permanecer constantes ou a se deteriorar. Desse modo, as curvas representativas dos resultados dos testes motores mostraram-se, em ambos os sexos, essencialmente ascendentes e paralelas até próximo ao início da adolescência. Depois, enquanto os resultados apresentados pelos rapazes induziram a uma elevação acentuada nas inclinações das curvas, sugerindo o aparecimento de um surto de desempenho motor nesse período, entre as raparigas as curvas tenderam a ser mais planas, oferecendo indícios de já terem alcançado o estágio adulto quanto ao desempenho motor. Nas tarefas motoras que exigem o deslocamento da própria massa corporal, as diferenças entre ambos os sexos tornaram-se progressivamente maiores com a idade e se acentuaram enormemente na adolescência.

Revendo alguns estudos disponibilizados na literatura, parece existir consenso quanto às dificuldades apresentadas pelas raparigas em demonstrar melhor desempenho motor ao longo dos anos na adolescência (Freitas et al., 2002; Guedes \& Guedes, 2002; Jürimäe \& Volbekiene, 1998; Malina, 2007). As implicações negativas associadas à estrutura e à composição corporal que surgem simultaneamente com a puberdade no sexo feminino podem, em parte, justificar a redução na capacidade de realização de determinadas tarefas motoras. Contudo, em tese, a deterioração do desempenho motor nas raparigas adolescentes não deve ser sustentada apenas com base em princípios biológicos. Existem fatores motivacionais de origem 
sociocultural que também podem contribuir para que as raparigas nas idades mais avançadas venham a apresentar limitações quanto ao alcance de melhor desempenho motor.

No campo especulativo, torna-se possível assumir que as implicações socioculturais associadas ao desempenho motor entre as raparigas possam se originar já na infância, ao se dispensar tratamento diferenciado por parte dos pais e da própria sociedade às crianças de um e de outro sexo por meio da seleção de ações motoras menos intensas para as raparigas, provocando de forma involuntária diferenças no tratamento de ambos os sexos desde as idades mais precoces. Na sequência, ao se ingressar na estrutura escolar, as eventuais diferenças ao tratar com os dois sexos tendem a aumentar em razão da sociedade e, particularmente, dos professores de educação física, que aceitam essas diferenças iniciais como unicamente de cunho biológico. Por sua vez, estes continuam a tratar as crianças de maneira diferenciada, não oferecendo atividades físicas semelhantes a ambos os sexos. Na adolescência, a menor motivação e disposição diante do desconforto induzindo pelo esforço físico e considerando o tradicional menor interesse na participação em programas sistematizados de exercício físico e de prática esportiva que procuram promover o desenvolvimento da capacidade de realizar movimento, impede que as raparigas possam aproveitar as vantagens biológicas induzidas pelo advento da puberdade na realização de determinadas tarefas motoras. Provavelmente, se em nossa realidade a sociedade brasileira procurasse valorizar a prática habitual de atividade física entre as raparigas, assim como o fazem em outros países social e culturalmente mais evoluídos, o comportamento do desempenho motor das raparigas poderia coincidir com o que é esperado biologicamente e, embora os escores equivalentes aos resultados dos testes motores venham a apresentar curvas com inclinação mais gradual, seria possível uma aproximação com a dos rapazes.

Quanto aos resultados observados por intermédio da administração do teste sit-andreach, em que é exigida a participação da flexibilidade de várias articulações simultaneamente, verificou-se que as raparigas demonstraram tendência a apresentar escores relativamente menores a cada ano, dos 6 aos 11 anos de idade e, na sequência, experimentaram aumento até os 18 anos. Os rapazes mostraram comportamento de declínio e ascensão similar ao observado nas raparigas; contudo, com ponto de inflexão dois anos mais tarde, aos 13 anos de idade.

De maneira similar ao que foi encontrado em outros estudos (Freitas et al., 2002; Guedes \& Guedes, 2002; Jürimäe \& Volbekiene, 1998; Malina, 2007), as raparigas, em todo o período etário considerado, apresentaram valores médios mais elevados que os rapazes, superioridade esta apontada estatisticamente dos 12 aos 14 anos de idade. As diferenças anatómicas e a maior aceitabilidade de atividades em que os movimentos de flexibilidade são enfatizados, em substituição aos mais vigorosos quanto à participação da força e da resistência muscular podem ter favorecido os resultados encontrados entre as raparigas. Quanto às diferenças anatómicas, uma vez que após a puberdade, os rapazes em geral apresentam maior comprimento de pernas proporcionalmente à estatura que as raparigas (Malina et al., 2004), parte da superioridade observada nos escores apresentados pelas raparigas talvez possa ser explicada pelas diferenças entre os sexos na morfologia esquelética, tendo em vista a sua aparente participação no resultado desse teste motor. Ainda, existe possibilidade da redução temporária dos escores observados nas raparigas até os 11 anos, e nos rapazes até os 13 anos, possa estar associada ao surto de crescimento pré-púbere, momento em que os ossos longos apresentam proporção de crescimento longitudinal mais acentuado que 
os músculos e os tendões, induzindo, nas crianças nessas idades, uma dificuldade temporária em se alongar até que seja alcançado o "catch-up" de crescimento dessas estruturas.

Relativamente aos resultados obtidos com a administração do teste curl-ups, em que existe o envolvimento da capacidade de força $\mathrm{e}$ resistência dos grupos musculares localizados na região inferior do tronco, verificou-se que, nas raparigas, os escores apresentaram discreto aumento dos 6 aos 12 anos de idade. Depois mostraram forte tendência a permanecer constantes até por volta dos 15 anos, quando iniciaram progressivo decréscimo até os 18 anos. Os rapazes, ao contrário, exibiram aumento bastante acentuado dos 6 aos 18 anos de idade. Na comparação dos valores médios observados entre os sexos, constatou-se que a superioridade apresentada pelos rapazes foi apontada em linguagem estatística a partir dos 13 anos e, com o passar dos anos, as diferenças aumentaram cada vez mais.

O melhor desempenho observado no teste curl-ups paralelamente ao avanço da idade, pode ser explicado pelo aumento simultâneo da força e da resistência muscular que ocorre naturalmente durante a infância e a adolescência em jovens de ambos os sexos (Malina et al., 2004). No entanto, o fato das raparigas alcançarem tão precocemente os escores mais elevados, seguido por uma tendência de nivelamento e declínio a partir dos 13 anos, conflituando, portanto, com a noção da evolução esperada de força e resistência muscular no sexo feminino nesse período, possivelmente pode ser explicado em razão da massa muscular entre as raparigas aumentar em proporção apenas moderada durante a puberdade, enquanto o acúmulo de gordura torna-se bastante acentuado nesse período, fundamentalmente na região dos quadris, dificultando sobremaneira a realização desse tipo de movimento.

No que se refere aos resultados encontrados por intermédio da administração do teste trunk-lift, tarefa motora em que a capacidade de flexibilidade é solicitada mediante movimento de elevação do tronco, estando o sujeito posicionado em decúbito ventral, os escores encontrados em ambos os sexos demonstraram comportamento ascendente até próximo dos 12 anos, oferecendo a impressão de certo paralelismo, com as raparigas apresentando valores médios discretamente mais elevados. $\mathrm{Na}$ sequência, raparigas e rapazes demonstraram ter alcançado um platô entre seus escores, portanto, as curvas representativas de cada sexo se dirigiram em uma mesma direção.

Confirmando a tendência observada nos resultados encontrados no teste sit-and-reach, em que a flexibilidade também é a principal capacidade física envolvida, as raparigas, em todo o período etário considerado, apresentaram valores médios equivalentes aos resultados do teste trunk-lift discretamente mais favoráveis que os rapazes. Estes achados corroboram com informações disponibilizadas na literatura (Freitas et al., 2002; Jürimäe \& Volbekiene, 1998; Guedes \& Guedes, 2002; Malina, 2007) e confirmam a vantagem das raparigas na realização de ações motoras que envolvem a flexibilidade. No entanto, uma diferença importante foi constatada no comportamento dos escores de ambos os testes motores. No caso do teste sit-and-reach, inicialmente, em ambos os sexos, verificou-se um declínio na magnitude dos escores, para somente a partir dos 11-13 anos apresentar valores proporcionalmente mais elevados com a idade; já no caso do teste trunk-lift, em todo o período etário considerado não se visualizou, em nenhum momento, tendência de declínio na magnitude dos escores equivalentes aos seus resultados. Provavelmente as tarefas motoras executadas especificamente em cada teste motor possam ter contribuído para que fosse observado comportamento diferenciado entre os escores equivalentes aos resultados de ambos os testes motores.

Os escores equivalentes aos resultados encontrados mediante a administração do teste push-ups, empregado como importante 
indicador para aferição das capacidades de força e resistência dos grupos musculares da região superior do tronco e dos membros superiores, entre os rapazes, apresentaram discreto aumento dos 6 aos 12 anos. Entretanto, após essa idade os valores se elevaram abruptamente até os 18 anos. Entre as raparigas, os escores demonstraram comportamento acentuadamente diferente, com valores bastante semelhantes dos 6 aos 18 anos; porém, com discreta tendência de declínio com a idade. Também não ocorreu nenhuma sequência de elevação, nem mesmo nas idades mais precoces, como ocorreu entre os resultados dos demais testes motores administrados no presente estudo.

$\mathrm{Na}$ comparação entre os valores médios apresentados por ambos os sexos, as diferenças observadas não se mostraram estatisticamente significantes até os 9 anos de idade, apesar de se constatar clara superioridade dos rapazes em relação às raparigas. Após essa idade, as diferenças se acentuaram ainda mais, recebendo destaque estatístico de tal forma que, aos 18 anos, os rapazes conseguiram executar, em média, quantidade de repetições quatro vezes maior que as raparigas. Neste sentido, enquanto os escores progressivamente maiores apresentados pelos rapazes a partir dos 13 anos podem ser justificados pelo fato de o advento da puberdade no sexo masculino induzir a um ganho de massa muscular bastante acentuado em consequência da maior produção de hormônios andrógenos, fazendo com que apresentem capacidade de força $e$ resistência mais elevada com a idade. O fato das raparigas não ter conseguido apresentar tendência evolutiva ao longo das idades deve ser examinado com alguma cautela.

Da mesma forma que os rapazes, entretanto de maneira menos acentuada, a maior produção dos hormônios femininos na puberdade deverá proporcionar importante ganho de massa muscular com a idade até próximo ao final da adolescência, o que também deverá induzir a maior capacidade de força e resistência muscular. Contudo, paralelamente ao aumento da massa muscular, deverá ocorrer também, um maior acúmulo de gordura corporal que, por sua vez, não deverá contribuir para a realização de movimentos que envolvem força e resistência muscular; no entanto, deverá traduzir-se em aumento importante da massa corporal (Malina et al., 2004). Sendo assim, embora seja possível detectar aumentos significativos no componente de força quando tratado em valores absolutos, isso já não deverá ocorrer necessariamente ao se envolver a massa corporal como fator de correção para a força das raparigas.

Consequentemente, uma razoável explicação para a identificação de um padrão constante dos 6 aos 18 anos de idade entre as raparigas, evidenciando desempenho semelhante durante todo esse período etário, pode estar alicerçada na natureza da tarefa motora exigida neste teste. A ação de push-ups envolve movimentos da própria massa corporal por meio de sua elevação e sustentação a uma determinada altura, caracterizada, portanto, por exigir maior solicitação do componente de força e resistência muscular em valores relativos que absolutos. Assim, revendo o padrão evolutivo esperado da força e da resistência muscular relativa das raparigas, constata-se que a vantagem observada com o aumento da massa muscular com a idade tende a diminuir, ou até mesmo a se anular, na medida em que a massa corporal apresenta valores mais elevados. Por isso, seus escores também não apresentam aumentos com a idade, apontando para um comportamento bastante próximo ao encontrado entre os resultados apresentados no presente estudo. Enquanto os rapazes alcançam valores máximos em força relativa somente na idade adulta, entre as raparigas o pico máximo da força relativa ocorre antes do início da puberdade, particularmente quando se trata do envolvimento dos grupos musculares da parte superior do tronco (Malina et al., 2004).

Quanto aos resultados do teste PACER, em que a capacidade cardiorrespiratória apresenta 
participação decisiva na execução da tarefa motora, os rapazes apresentaram escores continuamente mais elevados desde os 6 até os 18 anos. As raparigas, embora em proporção acentuadamente menor em comparação aos rapazes, também demonstraram valores crescentes; no entanto, apenas entre as idades de 6 e 13 anos, seguida por uma estabilização de alguns anos e encerrando com clara tendência de redução em seus valores. Ao se comparar os valores médios encontrados entre as raparigas e os rapazes, verificou-se que, juntamente com o teste de puxada em suspensão na barra modificada, esse foi o teste motor que apresentou as maiores discrepâncias entre ambos os sexos. Dos 6 aos 10 anos, as diferenças foram negligenciáveis; contudo, a partir dos 11 anos, a superioridade dos rapazes para caminhar/correr longas distâncias passou a ser evidenciada estatisticamente e, com o avanço das idades, essa diferença se acentuou cada vez mais.

Em busca de uma explicação plausível que possa justificar as acentuadas diferenças entre os sexos quanto ao desempenho em testes motores que envolve caminhada/corrida de longa distância, Thomas, Nelson e Church (1988) procuraram identificar a associação de seus escores com selecionados fatores biológicos e ambientais. Neste caso, foi constatado que o único fator biológico ao longo de toda a infância e a adolescência que está associado aos resultados de testes motores com essas características é a quantidade de gordura corporal, de tal modo que crianças e adolescentes com mais elevada quantidade de gordura corporal tende a percorrer longas distâncias mais lentamente. Contudo, ajustando-se o desempenho nos testes para a quantidade de gordura corporal, constatou-se que, se entre as crianças mais jovens foram observadas diferenças entre os sexos bastante reduzidas, quase inexistentes, durante e após a puberdade os resultados mostraram que, apesar de ocorrerem diferenças no desempenho dos testes de longa distância entre os sexos, estas se reduziram de forma bastante acentuada quando corrigidas pela quantidade de gordura corporal. Ainda assim, os rapazes continuaram a apresentar índices mais elevados, o que reflete provavelmente a influência de outros fatores biológicos além da quantidade de gordura corporal, supostamente maior comprimento de pernas, massa muscular mais desenvolvida e maior eficiência do sistema de produção de energia aeróbia. A par disso, admitindo-se que a quantidade de gordura corporal possa ser fortemente influenciada pela dieta e pelo nível de prática habitual de atividade física da criança e do adolescente, parece bastante provável que antes da puberdade as diferenças entre ambos os sexos no desempenho dos testes de caminhada/corrida de longa distância sejam induzidas por fatores ambientais e culturais, ao passo que após o início da puberdade alguns outros fatores biológicos devem também ser considerados.

Outro aspecto bastante interessante relacionado ao comportamento evolutivo e às diferenças entre os sexos quanto ao desempenho em testes motores que envolve caminhada/corrida de longa distância refere-se ao que se tem denominado de economia de corrida, originalmente "running economy". Nesse particular, Bar-Or (1984) observou que entre raparigas e rapazes de 5 a 17 anos de idade o consumo de oxigénio necessário para caminhar/correr em uma mesma velocidade decresce com a idade em ambos os sexos; porém, de forma mais acentuada entre os rapazes. Explicitamente, constatou-se que, deslocando-se a $10 \mathrm{~km} / \mathrm{h}$, o consumo de oxigénio de crianças aos 5 anos é, em média, 8 $\mathrm{mL} \cdot \mathrm{kg}^{-1} \cdot \mathrm{min}^{-1}$ maior que o de adolescentes aos 17 anos de idade. Dessa forma, considerando $40 \mathrm{~mL} \cdot \mathrm{kg}^{-1} \cdot \mathrm{min}^{-1}$ como o valor esperado para o consumo máximo de oxigénio nesse período etário, os adolescentes podem realizar a mesma tarefa motora com economia de aproximadamente $20 \%$ no consumo de oxigénio em comparação com as crianças.

Em outro estudo, Morgan, Martin e Krahenbuhl (1989) procuraram chamar a 
atenção para a possibilidade do menor gasto energético na realização de caminhadas/ corridas entre os adolescentes em comparação com as crianças não ser unicamente em consequência das diferenças observadas em seus respectivos metabolismos, destacando a maneira menos econômica de se locomover das crianças, tendo em vista a necessidade de uma frequência de passada mais elevada em razão do menor comprimento das pernas. $\mathrm{Na}$ comparação entre os sexos, fato semelhante também ocorreu com os rapazes, que se mostraram mais econômicos na tarefa motora de caminhar/correr que as raparigas. Por conseguinte, talvez a menor economia de corrida observada entre as crianças mais jovens possa explicar o fato do desempenho no teste PACER ficar tão distante daquela verificada na adolescência, considerando que ambos, crianças e adolescentes, apresentam valores de consumo de oxigénio bastante semelhantes (Payne \& Morrow, 1993), assim como a maior economia de corrida verificada entre os rapazes em relação as raparigas possa contribuir para que as diferenças entre os sexos nesse teste motor especificamente se acentuem ainda mais na adolescência.

\section{CONCLUSÕES}

Os achados encontrados revelaram que, nos cinco testes motores da bateria FitnessGram administrados no presente estudo, foram constatadas diferenças entre ambos os sexos desde os 6 até os 18 anos de idade. Contudo, nos testes motores em que são envolvidos os componentes de força e resistência muscular e resistência cardiorrespiratória, as diferenças inter-sexos aumentaram abruptamente a partir dos 12-13 anos de idade, favorecendo os resultados apresentados pelos rapazes.

Neste caso, em tese, os fatores biológicos que podem contribuir para que as diferenças no desempenho motor entre os sexos sejam tão claramente evidenciadas são sintetizados mediante: a) maior ganho de força muscular associado ao aumento na secreção de hormônios andrógenos na puberdade dos rapazes; b) maior acúmulo de gordura corporal nas raparigas próximo à puberdade; c) vantagem no tamanho corporal, traduzida pela medida de estatura, que ocorre nos rapazes após o surto puberal; d) vantagens anatômicas específicas dos rapazes, como maior comprimento das pernas e delineamento de quadris mais apropriado, beneficiando os sistemas de alavancas; e, e) vantagens nas funções fisiológicas nos rapazes, favorecendo a eficiência dos sistemas de produção e mobilização da energia biológica.

No que se refere ao comportamento dos escores em função da idade, se entre os rapazes a maioria dos testes motores administrados apresentou gradualmente melhores resultados até os 18 anos, entre as raparigas, os resultados mais elevados ocorreram por volta dos 11-12 anos e posteriormente tenderam a declinar ou a permanecer constantes. Supostamente, é possível inferir que as implicações negativas associadas à estrutura e à composição corporal que invariavelmente acompanham o advento da puberdade no sexo feminino, juntamente com atributos socioculturais adversos à prática habitual de atividade física, possam ter contribuído para que fossem observados decréscimos no desempenho motor de raparigas em idades mais avançadas.

\section{REFERÊNCIAS}

Allison, K., Adlaf, E., Dwyer, J., lysy, D., \& Irving, H. (2007). The decline in physical activity among adolescent students: A cross-national comparison. Canadian Journal of Public Health, 98(2), 97-110.

Bar-Or, O. (1984). Pediatric sports medicine for the practitioner: From physiologic principles to clinical applications. New York: Springer.

Bauman, A. E. (2004). Updating the evidence that physical activity is good for health: An epidemiological review 2000-2003. Journal of Science and Medicine in Sport, 7(S1), 6-19.

Cooper Institute for Aerobics Research - CIAR (1999). FitnessGram ${ }^{\circledR}$ test administration manual ( $2^{\text {nd }}$ ed.). Champaign, IL: Human Kinetics.

Fahlman, M., Hall, H., \& Lock, R. (2006). Ethnic and socioeconomic comparisons of fitness, 
activity levels, and barriers to exercise in high school females. Journal of School Health, 76(1), 12-17.

Fairclough, S. J., \& Stratton, G. (2006). A review of physical activity levels during elementary school physical education. Journal of Teaching in Physical Education, 25, 239-257.

Freitas, D., Maia, J., Marques, A., Beunen, G., Lefevre, J., Claessens, A., ... Philippaerts, R. (2002). Studies in somatic growth, biological maturation, physical fitness and activity in Portuguese speaking countries: An overview. Revista Portuguesa de Ciências do Desporto, 2(2), 92-111.

Gordon-Larsen, P., Nelson, M., \& Popkin, B. (2004). Longitudinal physical activity and sedentary behavior trends: Adolescence to adulthood. American Journal of Preventive Medicine, 27(4), 277-283.

Guedes, D. P., \& Guedes, J. E. (2002). Crescimento, composição corporal e desempenho motor de crianças e adolescentes. São Paulo: CLR Balieiro.

Guedes, D. P., \& Guedes, J. E. (2006). Manual prático para avaliação em educação física. São Paulo: Manole.

Hallal, P., Victora, C., Azevedo, M., \& Wells, J. (2006). Adolescent physical activity and health: A systematic review. Sports Medicine, 36(12), 1019-1930.

Hardy, L., Okely, A., Dobbins, T., \& Booth M. (2008). Physical activity among adolescents in New South Wales (Australia): 1997 and 2004. Medicine \& Science in Sports \& Exercise, 40(5), 835841.

Jürimäe, T., \& Volbekiene, V. (1998). Eurofit test results in Estonian and Lithuanian 11 to 17year-old children: A comparative study. European Journal of Physical Education, 3(3), 178-184.

Kim, J., Must, A., Fitamaurice, G., Gillman, M., Chomitz, V., Kramer, E., ... \& Peterson, K. (2005). Relationships of physical fitness to prevalence and incidence of overweight among schoolchildren. Obesity Review, 13(7), 12461254.

Lloyd, L., Bishop, P., Walker, J., Sharp, K., \& Richardson, M. T. (2003). The influence of body size and composition on FitnessGram test performance and the adjustment of FitnessGram test scores for skinfold thickness in youth. Measurement in Physical Education and Exercise Science, 7(3), 205-226.
Maia, J. A. R., \& Lopes, V. P. (2002). Estudo do crescimento somático, aptidão física, actividade física $e$ capacidade de coordenação corporal de crianças do primeiro ciclo do ensino básico da região autónoma dos Açores. Porto: Faculdade de Ciências do Desporto e de Educação Física - Universidade do Porto.

Malina, R. M. (2007). Physical fitness of children and adolescents in the United States: Status and secular change. Medicine and Sport Science, 50, 67-90.

Malina, R., Bouchard, C., \& Bar-Or, O. (2004). Growth, maturation, and physical activity. Champaign, IL: Human Kinetics.

Mastrangelo, M., Chaloupka, E., \& Rattigan, P. (2008). Cardiovascular fitness in obese versus no obese 8-11-year-old boys and girls. Research Quarterly for Exercise and Sport, 79 (4), 356-362.

Mondal, A. (2006). Physical and motor fitness level of Indian (Bengalee) school going girls. International Journal of Applied Sports Sciences, 18(2), 50-64.

Morgan, D., Martin, P., \& Krahenbuhl, G. (1989). Factors affecting running economy. Sports Medicine, 7(5), 310-330.

Mota, J., Flores, L., Ribeiro, J., \& Santos, M. (2006). Relationship of single measures of cardiorespiratory fitness and obesity in young schoolchildren. American Journal of Human Biology, 18(3), 335-341.

Nelson, M., Neumark-Stzainer, D., Hannan, P., Sirard, J., \& Story, M. (2006). Longitudinal and secular trends in physical activity and sedentary behavior during adolescence. Pediatrics, 118(6), 1627-1634.

Özdirenç, M., Özcan, A., Akin, F., \& Gelecek, N. (2005). Physical fitness in rural children compared with urban children in Turkey. Pediatrics International, 47(1), 26-31.

Payne, V. G., \& Morrow, J. R. (1993). Exercise and $\mathrm{VO}_{2}$ max in children: A meta-analysis. Research Quarterly for Exercise and Sport, 64 (4), 305-311.

Peña Reys, M., Tan, S., \& Malina, R. (2003). Urban rural contrasts in the physical fitness of school children in Oaxaca, México. American Journal of Human Biology, 15(6), 800-813.

Telama, R., Yang, X., Viikari, J., Välimäki, I., Wanne, O., \& Raitakari, O. (2005). Physical activity from childhood to adulthood: A 21-year tracking study. American Journal of Preventive Medicine, 28(3), 267-273. 
Thomas, J., Nelson, J., \& Church, G. (1988). A development analysis of gender differences in health related physical fitness. Phoenix: Arizona State University.
Tinazci, C., \& Emiroglu, O. (2009). Physical fitness of rural children compared with urban children in North Cyprus: A normative study. Journal of Physical Activity and Health, 6(1), 88-92.

(c) EY-Nc Todo o conteúdo da revista Motricidade está licenciado sob a Creative Commons, excepto quando especificado em contrário e nos conteúdos retirados de outras fontes bibliográficas. 